\title{
Wick Calculus For Nonlinear Gaussian Functionals
}

\author{
Yaozhong $\mathrm{Hu}^{*}$ and Jia-An $\mathrm{Yan}^{\dagger}$ \\ Department of Mathematics, University of Kansas \\ 405 Snow Hall, Lawrence, Kansas 66045-2142
}

\begin{abstract}
This paper surveys some results on Wick product and Wick renormalization. The framework is the abstract Wiener space. Some known results on Wick product and Wick renormalization in the white noise analysis framework are presented for classical random variables. Some conditions are described for random variables whose Wick product or whose renormalization are integrable random variables. Relevant results on multiple Wiener integrals, second quantization operator, Malliavin calculus and their relations with the Wick product and Wick renormalization are also briefly presented. A useful tool for Wick product is the $S$-transform which is also described without the introduction of generalized random variables.
\end{abstract}

Keyword: Malliavin calculus, Multiple integral, Chaos decomposition, Wick product, Wick renormalization

2000 MR Subject Classification: 60G15, 60H05, 60H07, 60H40

\section{Introduction}

In the constructive Euclidean quantum field theory, such as the $P(\phi)$ theory or the $: \phi^{4}$ : theory, there have been encountered infinite quantities, which originated (from mathematical point of view) from the product of generalized functions (see 23, [4, or [9] and the references therein for more details). To obtain useful information out of these infinite quantities, Wick (25) first introduced the now so-called Wick renormalization. According to [6, the Wick product in stochastic analysis was first introduced by Hida and Ikeda [5]. Meyer and Yan (21]) extended to cover the Wick products of Hida distributions. Now Wick

\footnotetext{
${ }^{*} \mathrm{Y}$. Hu was supported in part by the National Science Foundation under Grant No. DMS0504783, and the International Research Team on Complex Systems, Chines Academy of Sciences.

${ }^{\dagger}$ J.-A. Yan was supported by the National Natural Science Foundation of China (No. 10571167), the National Basic Research Program of China (973 Program) (No.2007CB814902), and the Science Fund for Creative Research Groups (No.10721101).
} 
product is applied to stochastic differential equations ([13]), stochastic partial differential equations, stochastic quantization ([9]) and many other fields.

In stochastic analysis, most of the research work on Wick product are on Hida distribution spaces or other spaces of generalized random variables. In this paper we survey some results that we have frequently used. To make the concept of Wick product accessible to broader audience, we restrict ourselves to the classical framework, namely, the classical random variables. In fact, a motivation to write such a survey is from some students who need to know some results relevant to Wick product and the way of how to use them in their research. After we have done some research on the references, we found out that many results on Wick product have been already known by the second author in the framework of Hida distribution ([26, 27]). But they are relatively unknown to the experts on the field.

In Section 2, we introduce the framework and some results which are useful in Wick product. In particular, we introduce the multiple integrals and the chaos expansion.

Malliavin calculus is very useful in the problems where Wick product presents. In Section 3, we give a simplest presentation of some results in Malliavin calculus which are relevant to wick product.

Wick product is introduced in Section 4. We present some basic results. Some of them may be new.

In Euclidean quantum field theory, the Wick renormalization is more widely used. In section 5, we present some results on Wick renomalization. In fact, in Euclidean quantum field theory a very special abstract Wiener space and a very special random variables are needed (see [9]). However, we will not go into detail.

This paper is a condensed survey of some results on Wick product. We do not intend to give a survey on the historical account. So in some cases, for a concept or a result, probably not the original references are going to be cited.

\section{Multiple integrals and chaos expansion}

Let $H$ be a separable Hilbert space with scalar product $\langle\cdot, \cdot\rangle_{H}$. There is a Banach space $B$ ( $B$ is not unique) with the following properties.

(i) $H$ is continuously embedded in $B$ and $H$ is dense in $B$. The dual $B^{\prime}$ (the space of continuous linear functionals) of $B$ is identified as a (dense) subspace of $H\left(B^{\prime} \subset B\right)$.

(ii) There is a Borel measure $\mu$ on $(B, \mathcal{B})$, where $\mathcal{B}$ is the Borel $\sigma$-algebra of $B$ such that

$$
\int_{B} \exp \{i\langle l, x\rangle\} d \mu(x)=\exp \left\{-\|l\|_{H}^{2} / 2\right\}, \quad l \in \Phi^{\prime},
$$

where $\langle\cdot, \cdot\rangle$ means the pairing between $B$ and $B^{\prime}$ (namely, $\langle l, x\rangle=l(x)$ ). 
The triple $(B, H, \mu)$ is an abstract Wiener space. We denote $\mathbb{E}(f)=\int_{B} f(x) d \mu(x)$ and $L^{p}=L^{p}(B, \mathcal{B}, \mu)$.

For any $l \in B^{\prime},\langle l, \cdot\rangle: B \rightarrow \mathbb{R}$ is a mean zero Gaussian random variable with variance $\|l\|_{H}^{2}$. By a limiting argument, for any $l \in H,\langle l, \cdot\rangle: B \rightarrow \mathbb{R}$ can be defined as a Gaussian random variable, denoted by $\tilde{l}$.

Fixed an $n$ and introduce the symmetric tensor product $H^{\hat{\otimes} n}$ by the following procedure.

Let $\left\{e_{1}, e_{2}, \cdots\right\}$ be an orthonormal basis of $H$ and let $\hat{\otimes}$ denote the symmetric tensor product. Then

$$
f_{n}=\sum_{\text {finite }} f_{i_{1}, \cdots, i_{n}} e_{i_{1}} \hat{\otimes} \cdots \hat{\otimes} e_{i_{n}}, \quad f_{i_{1}, \cdots, i_{n}} \in \mathbb{R}
$$

is an element of $H^{\hat{\otimes} n}$ with the Hilbert norm

$$
\left\|f_{n}\right\|_{H_{\otimes} \otimes}^{2}=\sum_{\text {finite }}\left|f_{i_{1}, \cdots, i_{n}}\right|^{2},
$$

$H^{\hat{\otimes} n}$ is the completion of all the elements of above form under the above norm.

To define the multiple integral, we need to use the Hermite polynomials. Let

$$
H_{n}(x)=(-1)^{n} e^{\frac{x^{2}}{2}} \frac{d^{n}}{d x^{n}} e^{-\frac{x^{2}}{2}}=\sum_{k \leq n / 2} \frac{(-1)^{k} n !}{2^{k} k !(n-2 k) !} x^{n-2 k}, \quad x \in \mathbb{R}
$$

be the $n$-th Hermite polynomial $(n=0,1,2, \cdots)$. Its generating function is

$$
e^{t x-\frac{t^{2}}{2}}=\sum_{n=0}^{\infty} \frac{t^{n}}{n !} H_{n}(x) .
$$

Any element $f_{n}$ in $H^{\hat{\otimes} n}$ of the form (2.2) can be rewritten as

$$
f_{n}=\sum_{\text {finite }} f_{j_{1}, \cdots, j_{m}} e_{j_{1}}^{\hat{\otimes} k_{1}} \hat{\otimes} \cdots \hat{\otimes} e_{j_{m}}^{\hat{\otimes} k_{m}}, \quad f_{j_{1}, \cdots, j_{m}} \in \mathbb{R},
$$

where $j_{1}, \cdots, j_{m}$ are different. For this type of integrands the multiple integral is defined as

$$
I_{n}\left(f_{n}\right):=\sum_{\text {finite }} f_{j_{1}, \cdots, j_{m}} H_{j_{1}}\left(\tilde{e}_{j_{1}}\right) \cdots H_{j_{m}}\left(\tilde{e}_{j_{m}}\right) .
$$

In particular, we have for $f \in H$

$$
I_{n}\left(f^{\otimes n}\right)=\|f\|_{H}^{n} H_{n}\left(\|f\|_{H}^{-1} \tilde{f}\right) .
$$

For general element $f_{n}$ in $H^{\hat{\otimes} n}$ we can define the multiple integral $I_{n}\left(f_{n}\right)$ by the $L^{2}$ convergence. It is straightforward to obtain the following isometry equality

$$
\mathbb{E}\left|I_{n}\left(f_{n}\right)\right|^{2}=n !\left\|f_{n}\right\|_{H^{\otimes} n}^{2} .
$$


One can also construct the Fock space $\Phi(H)$ on $H$ as follows.

$$
\Phi(H)=\bigoplus_{n=0}^{\infty} H^{\hat{\otimes} n} .
$$

The scalar product of two elements $f=\left(f_{0}, f_{1}, f_{2}, \cdots\right)$ and $g=\left(g_{0}, g_{1}, g_{2}, \cdots\right)$ in $\Phi(H)$ is defined as

$$
\langle f, g\rangle_{\Phi(H)}=\sum_{n=0}^{\infty} n !\left\langle f_{n}, g_{n}\right\rangle_{H^{\otimes} n} .
$$

The chaos expansion theorem states that any square integrable random variable $F$ on $(B, \mathcal{B}, \mu)$ can be written as

$$
F=\sum_{n=0}^{\infty} I_{n}\left(f_{n}\right), \quad f=\left(f_{0}, f_{1}, f_{2}, \cdots\right) \in \Phi(H)
$$

and

$$
\mathbb{E}\left(F^{2}\right)=\|f\|_{\Phi(H)}^{2}=\sum_{n=0}^{\infty} n !\left\|f_{n}\right\|_{H^{\otimes} n}^{2} .
$$

We refer to [20] and the references therein for further details.

Example 2.1 Let $B=H=\mathbb{R}^{d}$ and let $\mu$ be the standard Gaussian measure on $B$. Then $(B, \mathcal{B}, \mu)$ is the d-dimensional standard Gaussian measure space.

Example 2.2 Let

$$
H=\{f:[0, T] \rightarrow \mathbb{R} ; f(0)=0 \quad f \text { is absolutely continuous on }[0, T]\} .
$$

It is a Hilbert space under the norm $\langle f, g\rangle=\int_{0}^{T} f^{\prime}(t) g^{\prime}(t) d t$. Let

$$
\Omega=\{f:[0, T] \rightarrow \mathbb{R} ; f(0)=0 \quad f \text { is continuous on }[0, T]\}
$$

with the sup norm. Then $(\Omega, \mathcal{F}, \mu)$ is a canonical Wiener space, where $\mathcal{F}$ is the Borel $\sigma$-algebra on $\Omega$ (with respect to the sup norm).

Example 2.3 Consider a domain $D$ of $\mathbb{R}^{d}$ (d dimensional Euclidean space). Together with some nice boundary conditions (if $D$ is not the whole space $\mathbb{R}^{d}$ ) we can prove (see [4]) that there is a kernel $K(x, y)$ such that the following equation (with mass $m=1$ ) holds

$$
(-\Delta+1) K(x, y)=\delta(x-y),
$$

where $\Delta$ is the Dirichlet Laplacian on D. A Hilbert space of (generalized) functions can be determined by by

$$
\langle f, g\rangle=\int_{D} K(x, y) f(x) f(y) d x d y,
$$

where $f$ and $g$ are two (generalized) real-valued functions. The Gaussian measure associated with this Hilbert space is useful in the Euclidean quantum field theory (see also [9] and the references therein). 
Definition 2.4 Let $f_{n} \in H^{\hat{\otimes} n} \cdot f_{n}$ is called negative definite on $H^{\hat{\otimes} n}$ if

$$
\left\langle f_{n}, h^{\hat{\otimes} n}\right\rangle_{H^{\hat{\otimes} n}} \leq 0 \quad \forall h \in H
$$

Example 2.5 The following are proved in [9].

(i) If $f_{2} \in H^{\hat{\otimes} 2}$, then there is an $\alpha>0$ such that $\mathbb{E} \exp \left[\alpha I_{2}\left(f_{2}\right)\right]<\infty$. And $\mathbb{E} \exp \left[\alpha I_{2}\left(f_{2}\right)\right]<\infty$ is true for all $\alpha>0$ iff $f_{2}$ is negative definite (see [9], Theorem 5.1).

(ii) For any nonzero $f_{2 n+1}$ in $H^{\hat{\otimes} 2 n+1}(n \geq 1)$ and any $\lambda \in \mathbb{R}, \mathbb{E}\left(\exp \left\{\lambda I_{2 n+1}\left(f_{2 n+1}\right)\right\}\right)=$ $\infty$. (see [9], Theorem 5.2).

It is conjectured (see [9]) that

(iii) If $n$ is even, then $\mathbb{E} \exp \left[I_{n}\left(f_{n}\right)\right]<\infty$ iff $f_{n}$ is negative definite on $H^{\hat{\otimes} n}$.

Definition 2.6 If $F$ has a chaos expansion $F=\sum_{n=0}^{\infty} F_{n}$ and $\alpha \in \mathbb{R}$, then the second quantization operator of $\alpha$ acting on $F$ is defined as

$$
\Gamma(\alpha) F=\sum_{n=0}^{\infty} \alpha^{n} F_{n} .
$$

For this operator we have the following famous theorem.

Theorem 2.7 (Nelson's hypercontractivity) Let $1 \leq p<q<\infty$. The following inequality holds

$$
\|\Gamma(\alpha) F\|_{q} \leq\|F\|_{p}, \quad \forall F \in L^{p}
$$

if and only if $|\alpha| \leq \sqrt{\frac{p-1}{q-1}}$.

This inequality was first obtained by Nelson and appears in many places. See 2 , 7] and the references therein for further detail.

The following theorem is due to Üstünel and Zakai [24] and see [16] for a simpler proof.

Theorem 2.8 Let $f \in H^{\hat{\otimes} n}$ and $g \in H^{\hat{\otimes} m}$. Then $I_{n}(f)$ and $I_{m}(g)$ are independent if and only if

$$
\langle f, g\rangle_{H}=0,
$$

where $\langle f, g\rangle_{H} \in H^{\hat{\otimes} n+m-2}$ defined by

$$
\langle f, g\rangle_{H}=\sum_{n=1}^{\infty}\left\langle f, e_{n}\right\rangle_{H} \hat{\otimes}\left\langle g, e_{n}\right\rangle_{H}
$$

For an $f \in H^{\hat{\otimes} n}$ satisfying some more conditions on the existence of trace of $f$, the multiple Stratonovich integral $S_{n}(f)$ can also be introduced in the following way: 
Let $\left\{e_{1}, e_{2}, \ldots\right\}$ be an orthonormal basis of $H$. Let $f \in H^{\hat{\otimes} n}$ and consider the following random variable:

$$
S_{n}^{N}(f)=\sum_{k_{1}, \cdots, k_{n}=1}^{N}\left\langle f, e_{k_{1}} \hat{\otimes} \cdots \hat{\otimes} e_{k_{n}}\right\rangle_{H \hat{\otimes} n} \tilde{e}_{k_{1}} \cdots \tilde{e}_{k_{n}} .
$$

Definition 2.9 If as $N \rightarrow \infty, S_{n}^{N}(f)$ converges in $L^{2}$, then we say the multiple Stratonovich integral of $f$ exists. The limit is called the multiple Stratonovich integral of $f$ and is denoted by $S_{n}(f)$.

Definition 2.10 Denote

$$
\operatorname{Tr}^{k, N} f_{n}=\sum_{i_{1}, \cdots, i_{k}=1}^{N}\left\langle f, e_{i_{1}} \hat{\otimes} e_{i_{1}} \hat{\otimes} \cdots \hat{\otimes} e_{i_{k}} \hat{\otimes} e_{i_{k}}\right\rangle_{H \hat{\otimes} 2 k}
$$

which is considered as an element in $H^{\hat{\otimes}(n-2 k)}$. If as $N \rightarrow \infty, \operatorname{Tr}^{k, N} f_{n}$ converges in $H^{\hat{\otimes}(n-2 k)}$, then we say the trace of order $k$ exists and denote it by

$$
\operatorname{Tr}^{k} f=\sum_{i_{1}, \cdots, i_{k}=1}^{\infty}\left\langle f, e_{i_{1}} \hat{\otimes} e_{i_{1}} \hat{\otimes} \cdots \hat{\otimes} e_{i_{k}} \hat{\otimes} e_{i_{k}}\right\rangle_{H^{\hat{\otimes} 2 k}} .
$$

If the traces of order $k$ of $f$ exist for all $k \leq n / 2$, then the multiple Stratonovich integral of $f$, namely $S_{n}(f)$, exists and the following $\mathrm{Hu}$-Meyer formula holds

$$
\begin{aligned}
S_{n}(f) & =\sum_{k \leq n / 2} \frac{n !}{2^{k} k !(n-2 k) !} I_{n-2 k}\left(\operatorname{Tr}^{k} f\right) \\
I_{n}(f) & =\sum_{k \leq n / 2} \frac{(-1)^{k} n !}{2^{k} k !(n-2 k) !} S_{n-2 k}\left(\operatorname{Tr}^{k} f\right)
\end{aligned}
$$

For this result and other results see [1, [12, [15] and the references therein.

Example 2.11 If $f_{1}, \cdots, f_{n} \in H$, then

$$
S_{n}\left(f_{1} \hat{\otimes} f_{2} \hat{\otimes} \cdots \hat{\otimes} f_{n}\right)=\tilde{f}_{1} \tilde{f}_{2} \cdots \tilde{f}_{n} .
$$

\section{Malliavin calculus}

If $f_{n} \in H^{\hat{\otimes} n}$ and $g \in H$, then $\left\langle f_{n}, g\right\rangle_{H}$ is an element in $H^{\hat{\otimes}(n-1)}$. For $f_{n} \in H^{\hat{\otimes} n}$, we define

$$
D_{g} I_{n}\left(f_{n}\right)=n I_{n-1}\left(\left\langle f_{n}, g\right\rangle_{H}\right) .
$$

If for almost every $x \in B$, the above right hand is a continuous functional of $g$ on $H$, then

$$
D I_{n}\left(f_{n}\right)=n I_{n-1}\left(f_{n}\right)
$$


is a random variable with values in $H$. We can extend $D_{g}$ and $D$ to general random variable $F$ by linearity and limiting argument.

It is easy to check that $D(F G)=F D G+G D F$.

In the same way we can introduce higher order derivatives $D^{k} F: B \rightarrow H^{\hat{\otimes} k}$.

The space $D^{k, p}$ is defined as

$$
D^{k, p}=\left\{F: B \rightarrow \mathbb{R} ;\|F\|_{k, p}^{p}:=\sum_{i=0}^{k} \mathbb{E}\left\|D^{i} F\right\|_{H \hat{\otimes} i}^{p}<\infty\right\} .
$$

To describe this space, one may introduce the Ornstein-Uhlenbeck operator $L$ defined by

$$
L F=\sum_{n=1}^{\infty} n F_{n}, \quad \text { if } F \text { has the chaos expansion } \quad F=\sum_{n=1}^{\infty} F_{n}
$$

(which is the generator of the semigroup $P_{t} F=\Gamma\left(e^{-t}\right) F$ ).

The following result is called the Meyer's inequality (see [19] and also [22] for a simpler analytic proof).

Theorem 3.1 There is a constant $c_{k, p}$ and $C_{k, p}$ such that

$$
c_{k, p}\left\|(L+1)^{k / 2} F\right\|_{p} \leq\|F\|_{k, p} \leq C_{k, p}\left\|(L+1)^{k / 2} F\right\|_{p} .
$$

Meyer's inequality can be used to give a detailed description of $D^{k, p}$ even for non integer $k$.

Example 3.2 Let $f \in H$. Then

$$
\varepsilon(f):=\sum_{n=0}^{\infty} \frac{1}{n !} I_{n}\left(f^{\otimes n}\right)=\exp \left(\tilde{f}-\frac{1}{2}\|f\|_{H}^{2}\right)
$$

is called an exponential vector (in $L^{2}(B, \mathcal{B}, \mu)$ ).

For an exponential vector $\varepsilon(f)$ and $a g \in H$, we have

$$
D_{g} \varepsilon(f)=\varepsilon(f)\langle f, g\rangle, \quad D \varepsilon(f)=\varepsilon(f) f .
$$

Let

$$
\begin{gathered}
\mathcal{S}=\left\{F=h\left(\tilde{f}_{1}, \cdots, \tilde{f}_{n}\right), \quad f_{1}, \cdots, f_{n} \in H \quad \text { and } \quad h\right. \text { is smooth } \\
\text { function on } \left.\mathbb{R}^{n}, n \geq 1\right\} .
\end{gathered}
$$

Example 3.3 If $F=h\left(\tilde{f}_{1}, \cdots, \tilde{f}_{n}\right)$ is in $\mathcal{S}$ with $h$ being of polynomial growth, then $F \in D_{k, p}$ for all $k \geq 0$ and $p \geq 1$ and

$$
D_{g} F=\sum_{i=1}^{n} \frac{\partial h}{\partial x_{i}}\left(\tilde{f}_{1}, \cdots, \tilde{f}_{n}\right)\left\langle f_{i}, g\right\rangle
$$


and

$$
D F=\sum_{i=1}^{n} \frac{\partial h}{\partial x_{i}}\left(\tilde{f}_{1}, \cdots, \tilde{f}_{n}\right) f_{i} .
$$

Definition 3.4 If $F: B \rightarrow H$ and there is a random variable $Z$ such that

$$
\mathbb{E}(\langle F, D G\rangle)=\mathbb{E}(Z G) \quad \forall G \in \mathcal{S},
$$

Then we say the divergence of $F$ exists and we denote it by $Z=\delta(F)$.

This means that the divergence operator $\delta$ is the adjoint operator of the derivative operator $D$.

Example 3.5 If $g \in H$, then $\delta(g)=\tilde{g}$.

\section{$4 \quad$ Wick product}

Definition 4.1 If $f_{n} \in H^{\hat{\otimes} n}$ and $g_{m} \in H^{\hat{\otimes} m}$, then the Wick product of $I_{n}\left(f_{n}\right)$ and $I_{m}\left(g_{m}\right)$ is defined as

$$
I_{n}\left(f_{n}\right) \diamond I_{m}\left(g_{m}\right)=I_{n+m}\left(f_{n} \hat{\otimes} g_{m}\right),
$$

where $f_{n} \hat{\otimes} g_{m}$ denotes the symmetric tensor product of $f_{n}$ and $g_{m}$. If $F=$ $\sum_{n=0}^{N_{1}} I_{n}\left(f_{n}\right)$ and $G=\sum_{m=0}^{N_{2}} I_{m}\left(g_{m}\right)$, then we define

$$
F \diamond G=\sum_{n=0}^{N_{1}} \sum_{m=0}^{N_{2}} I_{n+m}\left(f_{n} \hat{\otimes} g_{m}\right),
$$

By a limiting argument, we can extend the Wick product to general random variables (see [20] and 9] for example).

Remark 4.2 Of course, when we use "by limiting argument" the definition depends on the topology that we use. We can approximate $F$ and $G$ by finite combination of multiple integrals, $F_{N}$ and $G_{N}$, and define $F \diamond G$ as the limit of $F_{N} \diamond G_{N}$. Different choices of the topology (for example, in probability, $L^{p}$, almost surely etc) will lead to different definitions of the Wick product. In this paper, we shall use the $L^{2}$ limit.

It is clear that $F, G \in L^{2}$ does not imply that $F \diamond G$ is a well-defined object in $L^{2}$. Now we present a sufficient condition on $F$ and $G$ such that $F \diamond G \in L^{2}$. To this end, we need to introduce some new norms.

If $F=\sum_{n=0}^{\infty} I_{n}\left(f_{n}\right)$, we define

$$
\|F\|_{(r)}^{2}=\|\Gamma(r) F\|^{2}=\sum_{n=0}^{\infty} n ! r^{2 n}\left\|f_{n}\right\|_{2}^{2} .
$$

The following proposition can be found in [27](Theorem 3.1). 
Proposition 4.3 Let $\frac{1}{p^{2}}+\frac{1}{q^{2}}=\frac{1}{r^{2}}, p, q, r>0$. Then

$$
\|F \diamond G\|_{(r)} \leq\|F\|_{(p)}\|G\|_{(q)}
$$

Proof Let $F=\sum_{n=0}^{\infty} I_{n}\left(f_{n}\right)$ and $G=\sum_{n=0}^{\infty} I_{n}\left(g_{n}\right)$. Denote $h_{n}=\sum_{k+j=n} f_{k} \hat{\otimes} g_{j}$. Let $a=r^{-2} q^{2}-1$. Then $1+a^{-1}=r^{-2} p^{2}$. We have

$$
\begin{aligned}
\sqrt{n !}\left\|h_{n}\right\| & \leq \sqrt{n !} \sum_{k+j=n}\left\|f_{k}\right\|\left\|g_{j}\right\|=\sum_{k+j=n}\left(\begin{array}{l}
n \\
k
\end{array}\right)^{1 / 2} \sqrt{k ! j !}\left\|f_{k}\right\|\left\|g_{j}\right\| \\
& \leq\left(\sum_{k+j=n}\left(\begin{array}{l}
n \\
k
\end{array}\right) a^{k}\right)^{1 / 2}\left(\sum_{k+j=n} a^{-k} k ! j !\left\|f_{k}\right\|^{2}\left\|g_{j}\right\|^{2}\right)^{1 / 2} \\
& =\left(\sum_{k+j=n}(1+a)^{n} a^{-k} k ! j !\left\|f_{k}\right\|^{2}\left\|g_{j}\right\|^{2}\right)^{1 / 2} \\
& =\left(\sum_{k+j=n}\left(1+a^{-1}\right)^{k} k !\left\|f_{k}\right\|^{2}(1+a)^{j} j !\left\|g_{j}\right\|^{2}\right)^{1 / 2}
\end{aligned}
$$

which implies the result.

As a direct consequence of the above proposition we obtain a condition on $F$ and $G$ such that $F \diamond G$ is in $L^{2}$.

Theorem 4.4 If $\Gamma(p) F, \Gamma(q) G$ are in $L^{2}$ with $\frac{1}{p^{2}}+\frac{1}{q^{2}}=1$, then $F \diamond G$ exists as an element in $L^{2}$.

A useful tool in studying the Wick product is the so-called $S$-transformation.

Definition 4.5 Let $F \in L^{p}$ for some $p>1$. Then for any $\xi \in H F(\cdot+\xi)$ : $B \rightarrow \mathbb{R}$ is well-defined integrable random variable. The following functional from $H$ to $\mathbb{R}$

$$
S(F)(\xi)=\mathbb{E}[F(\cdot+\xi)], \quad \forall \xi \in H .
$$

is called the $S$-transformation of $F$.

By Cameron-Martin theorem we have

$$
S(F)(\xi)=\mathbb{E}[F \varepsilon(\xi)], \quad \forall \xi \in H .
$$

Consequently, if $F=\sum_{n=0}^{\infty} I_{n}\left(f_{n}\right)$, then

$$
S(F)(\xi)=\sum_{n=0}^{\infty} n !\left\langle f_{n}, \xi^{\otimes n}\right\rangle, \quad \forall \xi \in H .
$$

This implies that

$$
S(F \diamond G)(\xi)=S(F)(\xi) S(G)(\xi)
$$


for suitable $F$ and $G$. For $f \in H$, since $\varepsilon(f)=\sum_{n=0}^{\infty} \frac{1}{n !} I_{n}\left(f^{\otimes n}\right)$, we have

$$
S \varepsilon(f)(\xi)=\exp \{\langle f, \xi\rangle\},
$$

and consequently

$$
\varepsilon(f) \diamond \varepsilon(g)=\varepsilon(f+g), \quad \forall f, g \in H .
$$

We refer to [14 [17, [18 and the references therein for more details in the framework of white noise analysis.

Proposition 4.6 If $f_{1}, f_{2} \in H$ are two unit vectors which are orthogonal, then

$$
H_{n}\left(\tilde{f}_{1}\right) \diamond H_{m}\left(\tilde{f}_{2}\right)=H_{n}\left(\tilde{f}_{1}\right) H_{m}\left(\tilde{f}_{2}\right) .
$$

Under some suitable condition on $F$ and $G$, we have

$$
\begin{aligned}
D_{g}(F \diamond G) & =D_{g} F \diamond G+F \diamond D_{g} G \\
D(F \diamond G) & =D F \diamond G+F \diamond D G
\end{aligned}
$$

The following proposition can be found in [27] (Theorem 5.5).

Proposition 4.7 If $g \in H, F \in L^{2}(B, \mathcal{B}, \mu)$ and if $D_{g} F$ exists and is in $L^{2}(B, \mathcal{B}, \mu)$, then $F \diamond \tilde{g}$ exists in $L^{1}(B, \mathcal{B}, \mu)$ and

$$
F \diamond \tilde{g}=F \tilde{g}-D_{g} F=F \tilde{g}-\langle D F, g\rangle_{H} .
$$

Proof Let

$$
\mathcal{E}=\left\{\sum_{\text {finite }} a_{i} e^{\tilde{f}_{i}}, \quad \text { where } \quad a_{i} \in \mathbb{R}, \quad f_{i} \in H\right\}
$$

Then $\mathcal{E}$ is dense in $L^{2}$. It is easy to see that (4.8) is true for all elements in $\mathcal{E}$ by (4.5). A density argument shows the proposition.

Proposition 4.8 If $g \in H, F \in L^{2}(B, \mathcal{B}, \mu)$ and if $D_{g} F$ exists and is in $L^{2}(B, \mathcal{B}, \mu)$, then $F \diamond \tilde{g}$ exists in $L^{1}(B, \mathcal{B}, \mu)$ and

$$
F \diamond \tilde{g}=\delta(F g) \text {. }
$$

Proof Let $G \in \mathcal{S}$. Then

$$
\begin{aligned}
\mathbb{E}((F \diamond g) G) & =\mathbb{E}\left(F G \tilde{g}-G\langle D F, g\rangle_{H}\right) \\
& =\mathbb{E}\left(\langle D(F G), g\rangle_{H}-\langle G D F, g\rangle_{H}\right) \\
& =\mathbb{E}\left(\langle F D G, g\rangle_{H}\right)=\mathbb{E}\left(\langle D G, F g\rangle_{H}\right)
\end{aligned}
$$

Since $G$ is arbitrary, we show the proposition.

The following proposition is used in [3] and [8]. 


\section{Proposition 4.9}

$$
\mathbb{E}[(F \diamond \tilde{f})(G \diamond \tilde{g})]=\mathbb{E}\left[F G\langle f, g\rangle_{H}+D_{g} F D_{f} G\right] .
$$

Proof

$$
\begin{aligned}
\mathbb{E}[(F \diamond \tilde{f})(G \diamond \tilde{g})] & =\mathbb{E}[\delta(F f)(G \diamond \tilde{g})]=\mathbb{E}\left[F D_{f}(G \diamond \tilde{g})\right] \\
& =\mathbb{E}\left[F\left(D_{f} G \diamond \tilde{g}+G \diamond D_{f} \tilde{g}\right)\right] \\
& =\mathbb{E}\left[F\left(D_{f} G \diamond \tilde{g}+G\langle f, g\rangle_{H}\right)\right] \\
& =\mathbb{E}\left[F \delta\left(D_{f} G g\right)+F G\langle f, g\rangle_{H}\right] \\
& =\mathbb{E}\left[F G\langle f, g\rangle_{H}+D_{g} F D_{f} G\right] .
\end{aligned}
$$

This is the proposition.

Let $F=\exp \left\{s \tilde{f}-s^{2}\|f\|^{2} / 2\right\}$ and $G=\exp \left\{t \tilde{g}-t^{2}\|g\|^{2} / 2\right\}$, where $s$ and $t$ are two arbitrary constants (we use $\|\cdot\|$ to denote $\|\cdot\|_{H}$ ). We have

$$
\begin{aligned}
F \diamond G & =\exp \left\{s \tilde{f}+t \tilde{g}-\|s f+t g\|^{2} / 2\right\} \\
& =\exp \left\{s \tilde{f}-s^{2}\|f\|^{2} / 2\right\} \exp \left\{t \tilde{g}-t^{2}\|g\|^{2} / 2\right\} \exp \{-s t\langle f, g\rangle\} \\
& =\sum_{i, j, p=0}^{\infty} s^{i} t^{j}(s t)^{p} \frac{(-1)^{p} I_{i}\left(f^{\otimes} i\right) I_{j}\left(g^{\otimes} j\right)\langle f, g\rangle^{p}}{i ! j ! p !} \\
& =\sum_{m, n=0}^{\infty} s^{m} t^{n} \sum_{p \leq m \wedge n} \frac{(-1)^{p} I_{m-p}\left(f^{\hat{\otimes} m-p}\right) I_{n-p}\left(g^{\hat{\otimes} n-p}\right)\langle f, g\rangle^{p}}{p !(n-p) !(m-p) !}
\end{aligned}
$$

On other hand, we have

$$
F \diamond G=\sum_{n, m=1}^{\infty} s^{m} t^{n} I_{m}\left(f^{\hat{\otimes} m}\right) \diamond I_{n}\left(g^{\hat{\otimes} n}\right) / m ! n ! .
$$

Comparing the coefficients of $s^{m} t^{n}$, we can write the above formula as

$$
I_{m}\left(f^{\hat{\otimes} m}\right) \diamond I_{n}\left(g^{\hat{\otimes} n}\right)=\sum_{p \leq m \wedge n} \frac{(-1)^{p} n ! m !\langle f, g\rangle^{p}}{p !(n-p) !(m-p) !} I_{m-p}\left(f^{\hat{\otimes} m-p}\right) I_{n-p}\left(g^{\hat{\otimes} n-p}\right) .
$$

By using the Malliavin derivative, the above formula can be written as

$$
I_{m}\left(f^{\hat{\otimes} m}\right) \diamond I_{n}\left(g^{\hat{\otimes} n}\right)=\sum_{p \leq m \wedge n} \frac{(-1)^{p}}{p !}\left\langle D^{p} I_{m}\left(f^{\hat{\otimes} m}\right), D^{p} I_{n}\left(g^{\hat{\otimes} n}\right)\right\rangle_{H \hat{\otimes} p},
$$

where $D^{p} F$ is identified as a mapping from $\Omega$ to $H^{\hat{\otimes} p}$. By the polarization technique, we have if $f_{m}$ and $g_{n}$ are continuous symmetric functions of $m$ and $n$-variables, then

$$
I_{m}\left(f_{m}\right) \diamond I_{n}\left(g_{n}\right)=\sum_{p \leq m \wedge n} \frac{(-1)^{p}}{p !}\left\langle D^{p} I_{m}\left(f_{m}\right), D^{p} I_{n}\left(g_{n}\right)\right\rangle_{H \hat{\otimes} p}
$$


In the same way we can obtain that

$$
I_{m}\left(f_{m}\right) I_{n}\left(g_{n}\right)=\sum_{p \leq m \wedge n} \frac{1}{p !}\left\langle D^{p} I_{m}\left(f_{m}\right), \diamond D^{p} I_{n}\left(g_{n}\right)\right\rangle_{H \hat{\otimes} p},
$$

where we consider $D^{p} I_{m}\left(f_{m}\right)$ and $D^{p} I_{n}\left(g_{n}\right)$ as two random variables with values in $H^{\hat{\otimes} p}$ and $\left\langle D^{p} I_{m}\left(f_{m}\right), \diamond D^{p} I_{n}\left(g_{n}\right)\right\rangle_{H \hat{\otimes} p}$ is the Wick scalar product. More precisely,

$$
\begin{aligned}
& \left\langle D^{p} I_{m}\left(f_{m}\right), \diamond D^{p} I_{n}\left(g_{n}\right)\right\rangle_{H \hat{\otimes} p} \\
= & \sum_{k_{1}, \cdots, k_{p}=1}^{\infty}\left\langle D^{p} I_{m}\left(f_{m}\right), e_{k_{1}} \hat{\otimes} \cdots \hat{\otimes} e_{k_{p}}\right\rangle_{H \hat{\otimes} p} \diamond\left\langle D^{p} I_{n}\left(g_{n}\right), e_{k_{1}} \hat{\otimes} \cdots \hat{\otimes} e_{k_{p}}\right\rangle_{H} \hat{\otimes} p \\
= & \sum_{k_{1}, \cdots, k_{p}=1}^{\infty} m(m-1) \cdots(m-p+1) n(n-1) \cdots(n-p+1) I_{m+n-2 p}(h)
\end{aligned}
$$

with

$$
h=\sum_{k_{1}, \cdots, k_{p}=1}^{\infty}\left\langle f_{n}, e_{k_{1}} \hat{\otimes} \cdots \hat{\otimes} e_{k_{p}}\right\rangle \hat{\otimes}\left\langle g_{n}, e_{k_{1}} \hat{\otimes} \cdots \hat{\otimes} e_{k_{p}}\right\rangle,
$$

where as before, $\left\{e_{1}, e_{2}, \cdots\right\}$ is an orthonormal basis of $H$.

We cab define $\left\langle D^{p} G, \diamond D^{p} G\right\rangle_{H^{\otimes}}$ in a similar way.

The above two formulas are for single chaos. We can use the linearity and limiting argument to show that

Theorem 4.10 If all the Malliavin derivatives of $F$ and $G$ exists and satisfy for $H=F$ and $H=G$

$$
\sum_{p=1}^{\infty} \frac{1}{p !}\left\|D^{p} H\right\|_{H \hat{\otimes} p}^{2}<\infty
$$

then $F \diamond G$ exists as an element in $L^{1}(\Omega, \mathcal{F}, P)$ and

$$
F \diamond G=\sum_{p=0}^{\infty} \frac{(-1)^{p}}{p !}\left\langle D^{p} F, D^{p} G\right\rangle_{H^{\hat{\otimes} p}} .
$$

We can also try to find conditions for the following identity to hold.

$$
F G=\sum_{p=0}^{\infty} \frac{1}{p !}\left\langle D^{p} F, \diamond D^{p} G\right\rangle_{H^{\hat{\otimes} p}} .
$$

Example 4.11 If $G=\tilde{g}$ for some $g \in H$, then from (4.14)

$$
F \diamond G=F \tilde{g}-\langle D F, g\rangle .
$$


Example 4.12 If $G=I_{2}\left(g_{2}\right)$ for some $g_{2} \in H^{\hat{\otimes} 2}$, then from (4.14)

$$
F \diamond G=F I_{2}\left(g_{2}\right)-2\left\langle D F, I_{1}\left(g_{2}\right)\right\rangle+\left\langle D^{2} F, g_{2}\right\rangle .
$$

The following proposition states if $F$ and $G$ are independent and if they are in single chaos form, then their Wick product and the usual product are the same.

Proposition 4.13 If $F=I_{n}\left(f_{n}\right)$ and $G=I_{m}\left(g_{m}\right)$ are independent, where $f_{n} \in H^{\hat{\otimes} n}$ and $g_{m} \in H^{\hat{\otimes} m}$, then

$$
F \diamond G=F G .
$$

Proof It is a direct consequence of (4.11) and Theorem 2.7.

From this proposition it is natural to conjecture that if $F$ and $G$ are independent, then $F \diamond G=F G$.

Denote by $\tau_{\xi} F$ the translation operator: $\tau_{\xi} F(\omega)=F(\omega+\xi), \omega \in B$. In the framework of white noise analysis, the following identities are from [27. It holds in our framework here under suitable conditions which are not made precise here.

$$
\begin{aligned}
\tau_{\xi} F & =[\varepsilon(\xi) F] \diamond \varepsilon(\xi) \\
\tau_{\xi}(F \diamond G) & =\left(\tau_{\xi} F\right) \diamond\left(\tau_{\xi} G\right) \\
D(F \diamond G) & =(D F) \diamond G+F \diamond(D G) .
\end{aligned}
$$

\section{Wick renormalization}

It is natural to define the Wick power

$$
F^{\diamond k}=\overbrace{F \diamond F \diamond \cdots \diamond F}^{k} .
$$

If $l \in H$, then

$$
\begin{aligned}
\tilde{l}^{\diamond n} & =I_{n}\left(l^{\hat{\otimes} n}\right)=\|l\|^{n} H_{n}\left(\frac{\tilde{l}}{\|l\|}\right) ; \\
\exp ^{\diamond}(\tilde{l}) & =\exp \left(\tilde{l}-\frac{\|l\|^{2}}{2}\right) .
\end{aligned}
$$

If $f: \mathbb{R}^{d} \rightarrow \mathbb{R}$ is a real valued entire function, then

$$
f(x)=\sum_{n=0}^{\infty} \sum_{n_{1}+\cdots+n_{d}=n} a_{n_{1}, \cdots, n_{d}} x^{n_{1}} \cdots x_{d}^{n_{d}} .
$$


Theorem 5.1 Let $X_{1}=\tilde{l}_{1}, \cdots, X_{d}=\tilde{l}_{d}$ be independent Gaussian random variables. If $f: \mathbb{R}^{d} \rightarrow \mathbb{R}$ is real entire function given above such that

$$
\sum_{n=0}^{\infty} \sum_{n_{1}+\cdots+n_{d}=n} n_{1} ! \cdots n_{d} ! a_{n_{1}, \cdots, n_{d}}\left\|X_{1}\right\|_{2}^{2 n_{1}} \cdots\left\|X_{d}\right\|_{2}^{2 n_{d}}<\infty
$$

then

$$
f^{\diamond}\left(X_{1}, \cdots, X_{d}\right)=\sum_{n=0}^{\infty} \sum_{n_{1}+\cdots+n_{d}=n} a_{n_{1}, \cdots, n_{d}} X_{1}^{\diamond n_{1}} \diamond \cdots \diamond X_{d}^{\diamond n_{d}}
$$

is well-defined as an element in $L^{2}$. Moreover,

$$
\mathbb{E}\left[f^{\diamond}\left(X_{1}, \cdots, X_{d}\right)\right]^{2}=\sum_{n=0}^{\infty} \sum_{n_{1}+\cdots+n_{d}=n} n_{1} ! \cdots n_{d} ! a_{n_{1}, \cdots, n_{d}}^{2}\left\|X_{1}\right\|_{2}^{2} \ldots\left\|X_{d}\right\|_{2}^{2} .
$$

Remark 5.2 (i) It is obvious that $X_{1}^{\diamond n_{1}} \diamond \cdots \diamond X_{d}^{\diamond n_{d}}$ in (5.3) can be replaced by $X_{1}^{\diamond n_{1}} \cdots X_{d}^{\diamond n_{d}}$.

(ii) We also denote

$$
: f\left(X_{1}, \cdots, X_{d}\right):=f^{\diamond}\left(X_{1}, \cdots, X_{d}\right)
$$

which is called the Wick ordering or Wick renormalization of $f\left(X_{1}, \cdots, X_{d}\right)$. A limiting argument can be used to discuss the case $d=\infty$.

(iii) Wick renormalization is studied in [26] in the framework of white noise analysis and with the use of scaling operator.

Proof It is easy to see that

$$
\mathbb{E}\left[X_{1}^{\diamond n_{1}} \diamond \cdots \diamond X_{d}^{\diamond n_{d}}\right]^{2}=n_{1} ! \cdots, n_{d} !\left\|X_{1}\right\|_{2}^{2 n_{1}} \cdots\left\|X_{d}\right\|_{2}^{2 n_{d}} .
$$

The theorem is proved by the orthogonality of each term in (5.3).

Theorem 5.3 Let $X=\left(X_{1}, \cdots, X_{d}\right)$ and $f$ be as in the previous theorem. Let $\left(Y_{1}, \cdots, Y_{d}\right)$ be independent copy of $X_{1}, \cdots, X_{d}\left(Y_{1}, \cdots, Y_{d}\right.$ and $X_{1}, \cdots, X_{d}$ are independent and have the same joint probability distribution). Then

$$
f^{\diamond}\left(X_{1}, \cdots, X_{d}\right)=\mathbb{E}\left[f\left(X_{1}+i Y_{1}, \cdots, X_{d}+i Y_{d}\right) \mid X_{1}, \cdots, X_{d}\right]
$$

Proof We have (denote $\sum=\sum_{n=0}^{\infty} \sum_{n_{1}+\cdots+n_{d}=n}$ )

$$
\begin{aligned}
\mathbb{E}[f(X+i Y) \mid X] & =\sum a_{n_{1}, \cdots, n_{d}} \mathbb{E}\left[\left(X_{1}+i Y_{1}\right)^{n_{1}} \cdots\left(X_{d}+i Y_{d}\right)^{n_{d}} \mid X_{1}, \cdots, X_{d}\right] \\
& =\sum a_{n_{1}, \cdots, n_{d}} \mathbb{E}\left[\left(X_{1}+i Y_{1}\right)^{n_{1}} \mid X_{1}\right] \cdots \mathbb{E}\left[\left(X_{d}+i Y_{d}\right)^{n_{d}} \mid X_{d}\right]
\end{aligned}
$$

Thus it suffices to show that for every $n \geq 1$,

$$
\mathbb{E}\left[\left(X_{1}+i Y_{1}\right)^{n} \mid X_{1}\right]=X_{1}^{\diamond n} .
$$


In fact, we have for all $t \in \mathbb{R}$,

$$
\begin{aligned}
\mathbb{E}\left[e^{t\left(X_{1}+i Y_{1}\right)} \mid X_{1}\right] & =e^{t X_{1}-\frac{1}{2}\left\|Y_{1}\right\|_{2}^{2}}=e^{t X_{1}-\frac{1}{2}\left\|X_{1}\right\|_{2}^{2}} \\
& =\sum_{n=0}^{\infty} \frac{t^{n}}{n !} X_{1}^{\diamond n} .
\end{aligned}
$$

Expanding the left hand side in term of $t^{n}$ and comparing the coefficients of $t^{n}$, we prove (5.6) and hence the theorem.

Let us first use Theorem 4.10 to compute : $e^{\lambda X^{2}}:$, where $X$ is a standard normal random variable.

Proposition 5.4 If $|\lambda|<1$, then the Wick renormalization of $e^{\frac{1}{2} \lambda X^{2}}$ exists in the sense of Theorem 5.1 and

$$
: e^{\frac{1}{2} \lambda X^{2}}:=\frac{1}{\sqrt{\lambda+1}} e^{\frac{\lambda}{2(\lambda+1)} X^{2}} .
$$

Proof First we have

$$
e^{\frac{1}{2} \lambda x^{2}}=\sum_{n=0}^{\infty} a_{n} x^{n}
$$

where

$$
a_{n}= \begin{cases}0 & \text { if } n=2 k+1 \\ \frac{\lambda^{k}}{2^{k} k !} & \text { if } n=2 k\end{cases}
$$

Thus (5.2) is equivalent to $|\lambda|<1$.

Let $Y$ be a standard normal independent of $X$. From Theorem 4.10, we have

$$
\begin{aligned}
: e^{\frac{1}{2} \lambda X^{2}}: & =\mathbb{E}\left[e^{\frac{1}{2} \lambda(X+i Y)^{2}} \mid X\right] \\
& =\mathbb{E}\left[e^{\frac{1}{2} \lambda X^{2}+i \lambda X Y-\frac{1}{2} \lambda Y^{2}} \mid X\right] \\
& =e^{\frac{1}{2} \lambda X^{2}} \frac{1}{\sqrt{2 \pi}} \int_{-\infty}^{\infty} e^{i \lambda X y-\frac{\lambda+1}{2} y^{2}} d y \\
& =\frac{1}{\sqrt{\lambda+1}} e^{\frac{\lambda}{2(\lambda+1)} X^{2}} .
\end{aligned}
$$

Example 5.5 Let $f \in H^{\hat{\otimes} 2}$ and consider : $\exp \left(\frac{1}{2} I_{2}(f)\right)$ :.

It is well-known that there is an orthonormal basis $\left\{e_{1}, e_{2}, \cdots\right\}$ of $H$ and $\alpha_{n}, n=$ $1,2, \cdots$ such that $\sum_{n=1}^{\infty} \lambda_{n}^{2}<\infty$ and

$$
f=\sum_{n=1}^{\infty} \lambda_{n} e_{n} \hat{\otimes} e_{n}
$$


Thus

$$
I_{2}(f)=\sum_{n=1}^{\infty} \lambda_{n}\left(\tilde{e}_{n}^{2}-1\right)
$$

Therefore

$$
\begin{aligned}
: \exp \left(\frac{1}{2} I_{2}(f)\right): & =\prod_{n=1}^{\infty}: e^{\frac{1}{2} \lambda_{n}\left(\tilde{e}_{n}^{2}-1\right)}: \\
& =\prod_{n=1}^{\infty} e^{-\frac{\lambda_{n}}{2}-\frac{1}{2} \log \left(\lambda_{n}+1\right)+\frac{\lambda_{n}}{2\left(\lambda_{n}+1\right)} \tilde{e}_{n}^{2}}
\end{aligned}
$$

Example 5.6 If we use the multiple Stratonovich integral, we have

$$
\begin{aligned}
: S_{n}\left(f_{1} \hat{\otimes} f_{2} \hat{\otimes} \cdots \hat{\otimes} f_{n}\right): & =: \tilde{f}_{1} \tilde{f}_{2} \cdots \tilde{f}_{n}: \\
& =\tilde{f}_{1} \diamond \tilde{f}_{2} \diamond \cdots \diamond \tilde{f}_{n} \\
& =I_{n}\left(f_{1} \hat{\otimes} f_{2} \hat{\otimes} \cdots \hat{\otimes} f_{n}\right) .
\end{aligned}
$$

Let $f \in H^{\hat{\otimes} n}$ have all traces of order $k, k \leq n / 2$ and let $S_{n}^{N}(f)$ be given by (2.14). Then

$$
\begin{aligned}
: S_{n}^{N}(f): & =\sum_{k_{1}, \cdots, k_{n}=1}^{N}\left\langle f, e_{k_{1}} \otimes \cdots \otimes e_{k_{n}}\right\rangle_{H^{\otimes}{ }_{n}} \tilde{e}_{k_{1}} \diamond \cdots \diamond \tilde{e}_{k_{n}} \\
& =I_{n}\left(\sum_{k_{1}, \cdots, k_{n}=1}^{N}\left\langle f, e_{k_{1}} \otimes \cdots \otimes e_{k_{n}}\right\rangle_{H \hat{\otimes}_{n}} e_{k_{1}} \otimes \cdots \otimes e_{k_{n}}\right) \\
& \rightarrow I_{n}(f) \quad\left(\text { in } L^{2}\right) .
\end{aligned}
$$

Thus we see that if existence of trace, we have if $f \in H^{\hat{\otimes} n}$ have all traces of order $k, k \leq n / 2$, then $S_{n}(f)$ exists as an element of $L^{2}$ and

$$
: S_{n}(f):=I_{n}(f) \text {. }
$$

Combining the above with the Hu-Meyer formula we have

Proposition 5.7 If $f \in H^{\hat{\otimes} n}$ have all traces of order $k, k \leq n / 2$, then

$$
: I_{n}(f):=\sum_{k \leq n / 2} \frac{(-1)^{k} n !}{2^{k} k !(n-2 k) !} I_{n-2 k}\left(\operatorname{Tr}^{k} f\right) \text {. }
$$

Proof From the Hu-Meyer formula (2.15), we have

$$
\begin{aligned}
: I_{n}(f): & =\sum_{k \leq n / 2} \frac{(-1)^{k} n !}{2^{k} k !(n-2 k) !}: S_{n-2 k}\left(\operatorname{Tr}^{k} f\right): \\
& =\sum_{k \leq n / 2} \frac{(-1)^{k} n !}{2^{k} k !(n-2 k) !} I_{n-2 k}\left(\operatorname{Tr}^{k} f\right) .
\end{aligned}
$$


It is also known in [26] that

$$
: F G:=(: F:) \diamond(: G:) .
$$

In Euclidean quantum field theory, the existence of interacting field leads to the exponential integrability problem of $S_{n}(f)$ for some very special $f$ on some specific abstract Wiener space. The Wick renormalization method reduces the problem to exponential integrability problem of $I_{n}(f)$. For more detailed discussion see 9 .

\section{References}

[1] Budhiraja, A. and Kallianpur, G. The generalized Hu-Meyer formula for random kernels. Appl. Math. Optim. 35 (1997), no. 2, 177-202.

[2] Dellacherie, C.; Meyer, P.-A.; Maisoneuve, B. Probabilités et potentiel. V.

[3] Duncan, T.e E.; Hu, Y. and Pasik-Duncan, B. Stochastic calculus for fractional Brownian motion. I. Theory. SIAM J. Control Optim. 38 (2000), no. $2,582-612$.

[4] Glimm, J. and Jaffe, A. Quantum physics. A functional integral point of view. Second edition. Springer, New York, 1987.

[5] Hida, T. and Ikeda, N. Analysis on Hilbert space with reproducing kernel arising from multiple Wiener integral. Proc. Fifth Berkeley Sympos. Math. Statist. and Probability (Berkeley, Calif., 1965/66). Vol. II: Contributions to Probability Theory, Part 1 pp. 117-143 Univ. California Press, 1967.

[6] Holden, H.; Øksendal, B.; Ubó e, J. and Zhang, T. Stochastic partial differential equations. A modeling, white noise functional approach. Probability and its Applications. Birkhä user Boston, 1996.

[7] Hu, Y. A unified approach to several inequalities for Gaussian and diffusion measures. Séminaire de Probabilités, XXXIV, 329-335. Lecture Notes in Math., 1729, Springer, 2000.

[8] $\mathrm{Hu}, \mathrm{Y}$. Integral transformations and anticipative calculus for fractional Brownian motions. Mem. Amer. Math. Soc. 175 (2005), no. 825.

[9] Hu, Y. and Kallianpur, G. Exponential integrability and application to stochastic quantization. Appl. Math. Optim. 37 (1998), no. 3, 295-353.

[10] Hu, Y. and Meyer, P.-A. Chaos de Wiener et intégrale de Feynman. Séminaire de Probabilités, XXII, 51-71, Lecture Notes in Math., 1321, Springer, 1988. 
[11] Hu, Y. and Meyer, P.-A. Sur les intgrales multiples de Stratonovitch. Séminaire de Probabilités, XXII, 72-81, Lecture Notes in Math., 1321, Springer, 1988.

[12] Hu, Y. and Meyer, P. A. On the approximation of multiple Stratonovich integrals. Stochastic processes, 141-147, Springer, New York, 1993.

[13] Hu, Y. and Øksendal, B. Wick approximation of quasilinear stochastic differential equations. Stochastic analysis and related topics, V (Silivri, 1994), 203-231, Progr. Probab., 38, Birkhäuser Boston, 1996.

[14] Huang, Z.Y. and Yan, J.-A. Introduction to Infinite Dimensional Stochastic Analysis. Mathematics and its Applications, 502. Kluwer Academic Publishers, Dordrecht; Science Press, Beijing, 2000.

[15] Johnson, G. W. and Kallianpur, G. Homogeneous chaos, $p$-forms, scaling and the Feynman integral. Trans. Amer. Math. Soc. 340 (1993), no. 2, 503548 .

[16] Kallenberg, O. On an independence criterion for multiple Wiener integrals. Ann. Probab. 19 (1991), no. 2, 483-485.

[17] Kondratiev, Y. G.; Streit, L.; Westerkamp, W. and Yan, J.-A. Generalized functions in infinite-dimensional analysis. Hiroshima Math. J. 28 (1998), no. $2,213-260$.

[18] Kondratiev, Y. G.; Leukert, P. and Streit, L. Wick calculus in Gaussian analysis. Acta Appl. Math. 44 (1996), no. 3, 269-294.

[19] Meyer, P. A. Transformations de Riesz pour les lois gaussiennes. Seminar on probability, XVIII, 179-193, Lecture Notes in Math., 1059, Springer, Berlin, 1984.

[20] Meyer, P.A. Quantum probability for probabilists. Lecture Notes in Mathematics, 1538. Springer, Berlin, 1993.

[21] Meyer, P.-A. and Yan, J.-A. Distributions sur l'espace de Wiener (suite) d'aprés I. Kubo et Y. Yokoi. Séminaire de Probabilités, XXIII, 382-392, Lecture Notes in Math., 1372, Springer, 1989.

[22] Pisier, G. Riesz transforms: a simpler analytic proof of P.-A. Meyer's inequality. Séminaire de Probabilités, XXII, 485-501, Lecture Notes in Math., 1321, Springer, 1988.

[23] Simon, B. The $P(\phi)_{2}$ Euclidean (quantum) field theory. Princeton Series in Physics. Princeton University Press, 1974.

[24] Ustunel, A. S. and Zakai, M. On independence and conditioning on Wiener space. Ann. Probab. 17 (1989), no. 4, 1441-1453. 
[25] Wick, G. C. The evaluation of the collision matrix. Physical Rev. (2) 80, (1950). 268-272.

[26] Yan, J.-A. Notes on the Wiener semigroup and renormalization. Séminaire de Probabilités, XXV, 79-94, Lecture Notes in Math., 1485, Springer, Berlin, 1991.

[27] Yan, J.-A. Products and transforms of white-noise functionals (in general setting). Appl. Math. Optim. 31 (1995), no. 2, 137-153. 